\title{
Multiphoton Intrapulse Interference 3: Probing Microscopic Chemical Environments
}

\author{
Johanna M. Dela Cruz, Igor Pastirk, Vadim V. Lozovoy, Katherine A. Walowicz, and \\ Marcos Dantus* \\ Department of Chemistry, Michigan State University, East Lansing, Michigan 48824
}

Received: July 23, 2003; In Final Form: September 3, 2003

\begin{abstract}
Phase modulation, through its influence on the probability of two-photon excitation at specific frequencies, is used to probe a molecule's microscopic chemical environment. The spectral phase required is designed according to the principles of multiphoton intrapulse interference. We show experimental results where phase modulation of 20 fs pulses from a titanium sapphire laser oscillator controls the intensity of two-photon induced fluorescence from the $\mathrm{pH}$-sensitive dye 8-hydroxypyrene-1,3,6-trisulfonic acid (HPTS) in solution. We show that the dependence of fluorescence yield on the phase can be utilized as a means of distinguishing molecules in different environments. Consequently, this method can be used to achieve selective multiphoton microscopy. To illustrate this capability, we show images where phase modulation was used to selectively excite HPTS in acidic or basic microscopic regions.
\end{abstract}

\section{Introduction}

This work represents a continuing effort from our group focused on the design of tailored femtosecond pulses to achieve control of nonlinear optical excitation in large molecules based on the concept of multiphoton intrapulse interference (MII). ${ }^{1-3}$ Our goal is to elucidate well-defined and reproducible pulse shapes that can be used to enhance or suppress particular nonlinear optical transitions in large molecules such as laser dyes and proteins in solution. In this paper, we demonstrate the use of MII to probe the local and microscopic environment of molecules by selective two-photon laser induced fluorescence (LIF).

There has been much interest in the use of tailored ultrafast laser pulses to control the excitation of large molecules in condensed phase. ${ }^{4-7}$ Coherent control of large molecules in the condensed phase has been considered very challenging because of the large numbers of intra- and intermolecular degrees of freedom involved. ${ }^{8}$ Further complications arise from the extremely fast homogeneous ( $<100 \mathrm{fs})$ and inhomogeneous $(<10$ fs) electronic dephasing in these systems. ${ }^{9}$ Many of these studies have been based on the concept of optimal pulse shaping, whereby a learning algorithm optimizes the spectral phase of the laser field. ${ }^{10-13}$ These methods have the advantage of convergence to an "optimal" pulse shape. However, because of the sensitivity of high-intensity multiphoton excitation to a large number of experimental parameters (for example, pulse intensity, saturation, transverse-mode quality, pulse bandwidth, and spectral phase which is affected by transmission through lenses and windows and reflection from dielectric mirrors), it is difficult to learn principles from these experiments that can be applied consistently to different samples in different laboratories.

The influence of phase on two-photon excitation was explored by Broers et al., who proposed that certain phase functions could focus the second harmonic spectrum in the frequency domain like a Fresnel lens focuses light in the spatial domain. They demonstrated this principle by controlling the two-photon

* To whom correspondence should be addressed. E-mail: dantus@msu.edu. excitation of $\mathrm{Rb}$ atoms. ${ }^{14}$ Meshulach et al. used the same principle to demonstrate coherent control on the two-photon excitation of Cs atoms. ${ }^{15}$ Their work focused on the narrow two-photon absorption resonance; they showed that the coherent two-photon enhancement was absent in the broadband excitation of large molecules in solution. ${ }^{15}$ Work from our group has demonstrated that the changes introduced by pulse shaping on two- and three-photon transitions arise from destructive interference and not on focusing in the frequency domain. ${ }^{2}$ The theoretical framework developed by our group went beyond the narrow resonance condition and allowed us to develop MII for controlling multiphoton excitation in large molecules in solution. ${ }^{1,2}$ The fundamental advantage of our work was the early realization that very short pulses, with concomitant large bandwidths, would be needed to control the nonlinear excitation of complex systems. We realized that in the condensed phase inhomogeneous broadening would prevent the coherent manipulation of vibronic levels, as can be done in the gas phase. Therefore, we developed a method to control the delivery of energy to the molecule that was less dependent on intramolecular dynamics. MII has now proven to be a robust method that is amenable to a number of applications. ${ }^{3}$

The first application of phase tailored pulses for sensing $\mathrm{pH}$ involved intense linearly chirped laser pulses that saturated the one-photon resonant transition of a $\mathrm{pH}$-sensitive dye. ${ }^{16}$ The ratio between the LIF signal obtained for positively and negatively chirped pulses was found to vary from 1.9 to 1.2 for different $\mathrm{pH}$ values. However, this ratio did not vary monotonically, being the same for $\mathrm{pH} 7$ and $\mathrm{pH} 9$.

The method presented here is completely different from the early experiments of Buist et al. ${ }^{16}$ Our method involves very weak pulses inducing two-photon transitions. The samples are thus transparent at the carrier wavelength of the pulse, the transitions are not saturated, and the results are independent of probe molecule concentration. The mechanism we used for $\mathrm{pH}$ probing is understood and theoretical simulations are in close agreement with the data.

The probability for two-photon excitation, $S^{(2)}$, using phasemodulated pulses at frequency $\omega=\Delta+\omega_{0}$, where $\omega_{0}$ is the 


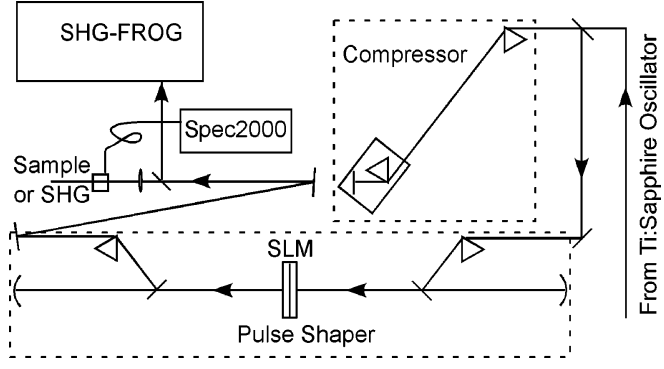

Figure 1. Schematic of the laser setup for MII experiments. The output of the titanium sapphire oscillator is compressed by the prism pair compressor before arriving at the pulse shaper. Phase and amplitude shaping take place at the spatial light modulator (SLM) which incorporates a polarization optic at its input and output windows. Once shaped, the pulses are directed toward the experimental cell. Characterization of the pulses by MIIPS requires replacing the sample cell by a thin SHG crystal; alternatively, the pulses are directed to an SHGFROG.

carrier frequency, can be calculated from the expression ${ }^{2}$

$$
S^{(2)} \propto \int g^{(2)}(2 \omega)\left|\int E(\omega+\Omega) E(\omega-\Omega) \mathrm{d} \Omega\right|^{2} \mathrm{~d} \omega
$$

where $E(\omega)=|E(\omega)| \mathrm{e}^{i \phi(\omega)}$ is the electric field of the laser. In this expression, we assume a broad nonlinear two-photon absorption spectrum, $g^{(2)}(2 \omega)$, and ignore intramolecular dynamics to simulate our experimental results. Consequences of this assumption are addressed later.

Here we demonstrate how the MII method, which couples the sensitivity of multiphoton excitation on the spectral phase of the laser pulses is used to probe microscopic chemical environment-induced changes in reporter molecules. We carry out the optimization of the required phase functions in solution and provide theoretical simulations. We show experimental images whereby $\mathrm{pH}$-selective two-photon microscopy is achieved and demonstrate how selective excitation can be used to enhance contrast and, consequently, to achieve functional imaging, using fluorescent probes sensitive to changes in their local environment.

\section{Experimental Section}

Experiments were performed using a titanium sapphire laser oscillator (K\&M Labs) capable of producing pulses with bandwidths up to $80 \mathrm{~nm}$ fwhm. The output of the oscillator was evaluated to make sure there were no changes in the spectrum across the beam and was compressed with a double prism pair arrangement shown in Figure 1. The pulse shaper uses prisms as the dispersive elements, two cylindrical concave mirrors, and a spatial light modulator (CRI Inc. SLM-256), composed of two 128-pixel liquid crystal masks in series. The SLM was placed at the Fourier plane. ${ }^{17}$ Because the retardance introduced by the liquid crystal is frequency dependent, pulse shaping of ultrashort pulses (with $>100 \mathrm{~nm}$ total bandwidth) requires calibration of each pixel element. At the end of the calibration, our data acquisition program maps the spectrum of our laser on the SLM in a frequency rather than wavelength scale. An algorithm has been programmed to independently control amplitude and phase at each frequency with the SLM. This allows us to define a Gaussian shape or spectrum in the frequency domain using an amplitude mask and then to scan the phase function without affecting the spectrum of the pulse. After compression and pulse shaping, $\sim 200 \mathrm{pJ}$ pulses were used to interrogate the samples.

The reproducibility of pulse shaping experiments depends on accurate characterization of the phase of the laser pulses. For this task, we use a single beam method, called multiphoton intrapulse interference phase scan (MIIPS), ${ }^{18}$ to measure both the sign and magnitude of phase distortions in the pulse. In MIIPS, a well-known reference function is scanned across the spectrum of the pulse to reveal the unknown phase across the pulse. For each phase in the scan, the second harmonic spectrum obtained from a thin $0.015 \mathrm{~mm} \beta \mathrm{BBO}$ crystal is recorded. Changes in the SHG spectrum are then used to obtain the spectral phase of the laser pulses. The MIIPS measurement takes place at the location of the sample; therefore, the exact phase at the sample is obtained.

For the experiments in this study, we started with very broad bandwidth pulses ( $~ 80 \mathrm{~nm}$ fwhm). We then defined Gaussian spectra of the desired width and center wavelength by amplitude shaping. Second, we measured the quadratic and cubic phase distortions in the pulse by scanning a phase function defined by

$$
f(\Delta)=\alpha \cos (\gamma \Delta-\delta)
$$

where the amplitude $\alpha$ was set to $1.5 \pi \mathrm{rad}, \gamma$ was set to $20 \mathrm{fs}$ (approximately the pulse duration), $\Delta$ is the frequency detuning from the center of the pulse, and $\delta$ is a parameter that determines the position of the maximum retardance (or the relative position of superimposed phase function). The MIIPS data is a twodimensional collection of SHG spectra as a function of $\delta$ (as shown in Figure 2). For transform-limited pulses, the MIIPS data shows equally spaced parallel linear features given by

$$
\delta_{\mathrm{TLmax}}(\lambda)=\left(\lambda-\lambda_{0}\right) \gamma \omega_{0}{ }^{2} / \pi c \pm \pi / 2
$$

deviations from these parallel lines indicate distortions in the spectral phase. For example, the spacing between the lines depends linearly on quadratic chirp according to

$$
\phi^{\prime \prime}=\alpha \gamma^{2}\left(x_{1}-x_{2}\right) / 4
$$

where $x_{1}-x_{2}$ is the difference between the distances of the lines (see Figure 2a,b). The cubic phase modulation is determined by the slope $\mathrm{d} \delta / \mathrm{d} \lambda$ that the maximum SHG features make in the $\lambda-\delta$ plane. Cubic phase modulation is given by

$$
\phi^{\prime \prime \prime}=\alpha \gamma^{2} \pi c / \omega_{0}^{2}\left[(\mathrm{~d} \delta / \mathrm{d} \lambda)_{1}-(\mathrm{d} \delta / \mathrm{d} \lambda)_{2}\right]
$$

where the slopes $\mathrm{d} \delta / \mathrm{d} \lambda$ are measured in $\mathrm{rad} / \mathrm{nm}$ (see Figure $2 \mathrm{a}, \mathrm{b})$. The expression required to retrieve arbitrary spectral phases, not only the quadratic and cubic components, is given elsewhere. ${ }^{18}$

The third step involves correction for phase distortions to yield transform-limited pulses. The spectral phase of the pulses $\phi(\lambda)$ was determined, and its complement, $-\phi(\lambda)$, was introduced in the shaper to render transform-limited pulses. When a subsequent MIIPS scan reveals a residual phase deformation (by deviation from the equidistant parallel lines required for transform-limited pulses), a second or third measurement and compensation iterations are required to obtain transform-limited pulses. The MIIPS of compensated pulses is shown in Figure 2b. To corroborate phase information retrieved from MIIPS, phase characterization was also done using second harmonic generation frequency resolved optical gating (SHG-FROG). ${ }^{19}$ SHG-FROG traces of the pulses before and after compensation are shown in Figure 2c,d. We found that, for small phase distortions (quadratic phase less than $200 \mathrm{fs}^{2}$ and cubic phase 

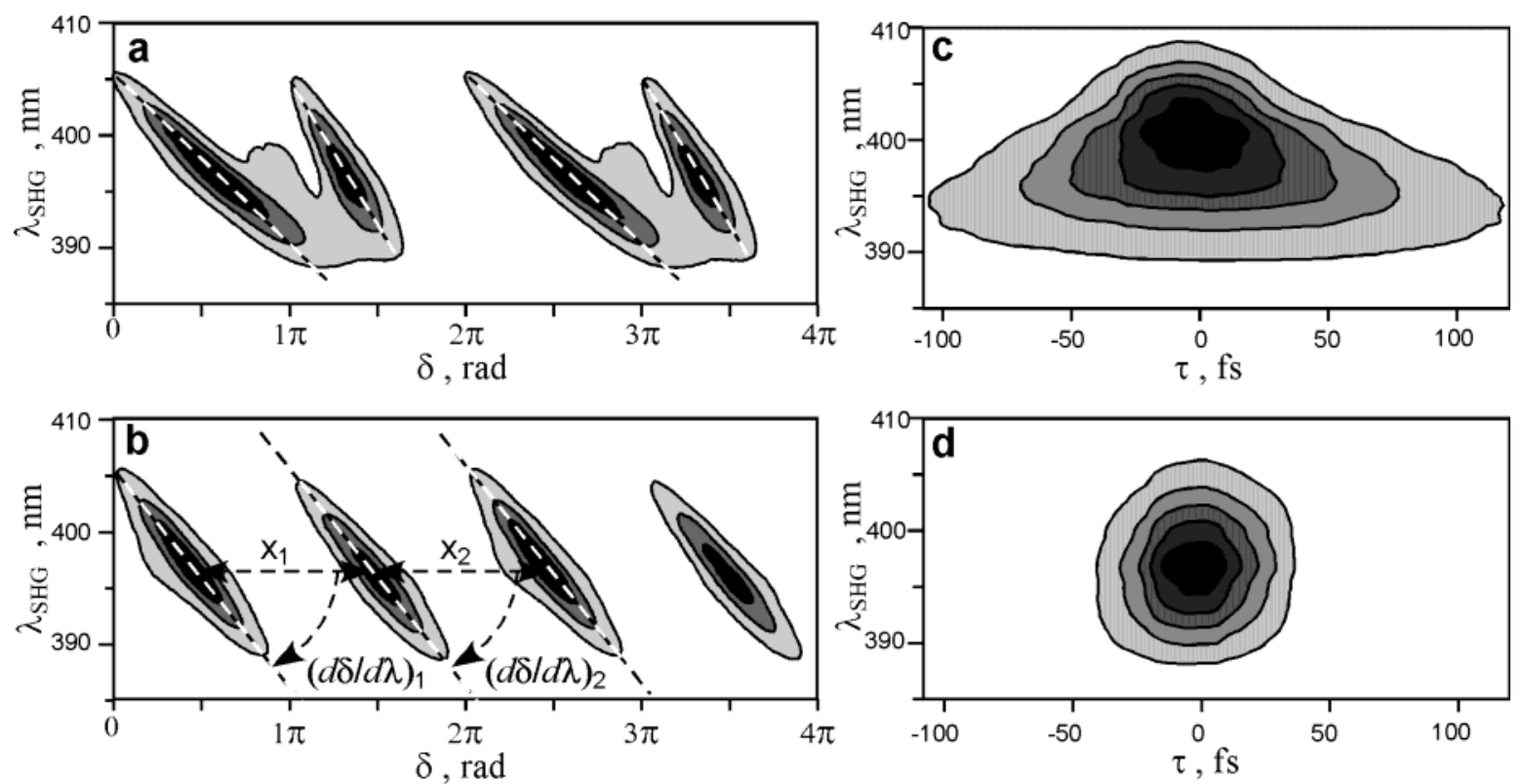

Figure 2. Compensation of spectral phase modulation in the femtosecond pulses. Spectral phase modulation in the femtosecond pulses is characterized (a) and compensated (b) using the MIIPS method. Quadratic phase distortions are determined by differences in the distances between the SHG features in the MIIPS scans. Cubic phase distortions are determined by differences between the slopes of the SHG features. Notice that after compensation differences in the distances and slopes (panel a) are greatly diminished (panel b). SHG-FROG traces for the pulses in panels a and $\mathrm{b}$ are shown in panels $\mathrm{c}$ and d. Note that the SHG-FROG trace becomes circular. The pulses before compensation had $-1000 \mathrm{fs}^{2}$ and $-19750 \mathrm{fs}^{3}$ of quadratic and cubic spectral phase, after compensation they were $5 \pm 20 \mathrm{fs}^{2}$ and $42 \pm 100 \mathrm{fs}^{3}$.

less than $1000 \mathrm{fs}^{3}$ ), MIIPS was more accurate and reproducible (down to $20 \mathrm{fs}^{2}$ and $100 \mathrm{fs}^{3}$ ) or $0.1 \mathrm{rad}$ across the spectrum of the pulses.

The final pulse shaping step involved the controlled phase modulation of transform-limited pulses. This step involved introducing phase functions determined by eq 2 . The laser was focused by a $50 \mathrm{~mm}$ focal length lens onto a $10 \mathrm{~mm}$ path length quartz cell containing the dye solution. Fluorescence perpendicular to the excitation beam was collected near the front face of the cell to minimize dispersion in the sample. We took advantage of the narrow acceptance angle of the optical fiber for spatial filtering and imaged the signal onto an Ocean Optics spectrometer (S2000). The experiment involved scanning the parameter $\delta$ and detection of the fluorescence of the sample (spectrally integrated). Signal integration time was typically 500 ms per spectrum for each value of $\delta$, and the data scan, with 256 different phase positions, was averaged over 3 cycles of $\delta$ from 0 to $4 \pi \mathrm{rad}$.

The samples used were $10^{-5} \mathrm{M}$ aqueous solutions of 8-hydroxypyrene-1,3,6-trisulfonic acid trisodium salt (HPTS or $3 \mathrm{sPyOH})$ (Sigma-Aldrich) in different $\mathrm{pH}$ buffers. The $\mathrm{pH} 6$ buffer solution was prepared from $0.1 \mathrm{M} \mathrm{KH}_{2} \mathrm{PO}_{4}(250 \mathrm{~mL})$ in $0.2 \mathrm{M} \mathrm{NaOH}$ (14 mL); $\mathrm{pH} 8$ buffer was prepared from the same solutions but with more $(115 \mathrm{~mL})$ of the $\mathrm{NaOH}$; whereas the pH 10 buffer solution was prepared from $0.025 \mathrm{M} \mathrm{Na}_{2} \mathrm{~B}_{4} \mathrm{O}_{7}$ $(250 \mathrm{~mL})$ in $0.2 \mathrm{M} \mathrm{NaOH}(27 \mathrm{~mL})$. The buffer solutions were diluted to $500 \mathrm{~mL}$ with distilled water. UV-vis absorption spectra of the dye solutions at different $\mathrm{pH}$ environments were obtained with a Hitachi U-4001 UV-vis spectrophotometer.

For the imaging experiment, shaped laser pulses, with an energy of $0.2-1 \mathrm{~nJ}$ per pulse and $87 \mathrm{MHz}$ repetition rate, were mildly focused to a spot size of $\sim 200$ microns in diameter on the sample. Two-photon induced fluorescence was collected by an infinity corrected apochromatic microscope objective (APO 100X, Mitutoyo) and imaged into a liquid-nitrogen-cooled CCD detector using an InfiniTube (Proximity Series, Infinity PhotoOptical). The sample was prepared by mixing $1 \mathrm{~mL}$ of acrylonitrile-vinylidene chloride polymer with $100 \mu \mathrm{L}$ of $10^{-2}$ $\mathrm{M}$ aqueous HPTS. For the acidic medium (pH 6), $20 \mu \mathrm{L}$ of 1 $\mathrm{M} \mathrm{HCl}$ was added to the HPTS-polymer mixture, and for the basic medium ( $\mathrm{pH} 10$ ), $140 \mu \mathrm{L}$ of $1 \mathrm{M} \mathrm{NaOH}$ was added. The $\mathrm{pH}$ of each mixture was checked; both mixtures were then smeared to an approximate thickness of $0.07 \pm 0.03 \mathrm{~mm}$ on contiguous regions of a microscope glass slide and then allowed to cure for $20 \mathrm{~min}$. Some mixing of the solvent water may have occurred at the interface between the two regions. Local changes in the fluorescence intensity observed for transform-limited pulses may reflect changes in the local sample thickness. The images were captured without optical filters in the collection optics or post image manipulation; they are shown in false color (both regions fluoresce at $515 \mathrm{~nm}$ ). The background counts, obtained when the laser is off, were subtracted.

\section{Results}

In Figure 3, we show the dependence of two-photon LIF on phase modulation. These measurements were obtained using 23 fs transform-limited pulses that were shaped to have a Gaussian spectrum centered at $842.6 \mathrm{~nm}$ with a bandwidth of $48 \mathrm{~nm}$ fwhm and spectral phase defined by eq 2 . Figure 3 a shows the experimental two-photon LIF intensity from HPTS in a pH 10 solution (dots) as a function of $\delta$. The signal shows a sinusoidal modulation. Figure $3 b$ shows the experimental two-photon LIF intensity from HPTS in a pH 6 solution (dots) as a function of $\delta$. The signal is modulated, however, and there is a clear phase shift compared to $\mathrm{pH} 10$. The shift comes about from $\mathrm{pH}-$ induced changes in the two-photon absorption spectrum. Experiments obtained for $\mathrm{pH} 8$ were similar to those obtained at $\mathrm{pH}$ 10 , but they contained a small shift that made them distinguishable. It is crucial to underline that the laser spectrum and intensity remain unchanged throughout the experiments and that the emission spectrum from the dye is independent of $\mathrm{pH}$.

Simulations of the experimental signal were performed using eq 1 without adjustable parameters. The spectrum of the pulse and the absorption spectrum of HPTS were measured experi- 


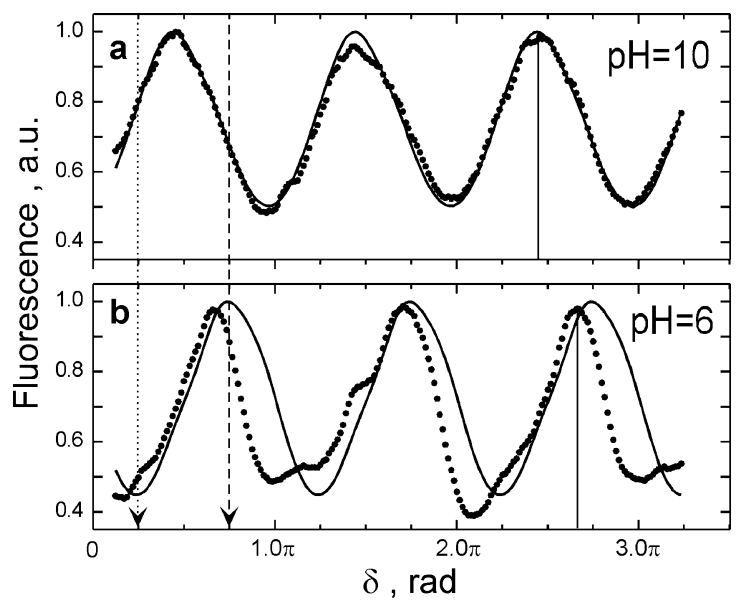

Figure 3. Experimental MII results and simulations for HPTS in basic and acidic solutions. Intensity of the two-photon induced fluorescence of HPTS solution at $\mathrm{pH} 10$ (a) and $\mathrm{pH} 6$ (b) are plotted as a function of the phase parameter $\delta$, according to eq 2. Simulations (continuous lines) based on eq 1 are in good agreement with the experimental data (dots). The arrows at $\delta=1 / 4 \pi$ (dotted) and $\delta=3 / 4 \pi$ (dashed) represent phase functions for which the intensity of two-photon fluorescence from HPTS at $\mathrm{pH} 10$ is similar but shows large differences for $\mathrm{pH} 6$. The optimum two-photon excitation of HPTS is indicated by vertical lines for the acid and basic solutions.

mentally. An examination of the molecular structure of HPTS shows that it has no center of symmetry. Because parity restrictions may be relaxed in this case, the similarity between one-photon and two-photon absorption spectra is expected. The spectral phase $f(\Delta)$ imprinted by the phase mask was the same used for the simulations. Both experimental and theoretical data were normalized such that the signal intensity is unity and the background observed is zero. The experimental data (dots) generally agree with the calculated response (continuous line) of the dyes in all $\mathrm{pH}$ environments (see Figure 3). Differences found for $\mathrm{pH} 6$ are discussed below.

We repeated our experiments with 50 fs pulses centered at $760 \mathrm{~nm}$. No differences in phase dependence were observed for the three different $\mathrm{pH}$ solutions, as expected and as corroborated by simulation. The absorption spectrum of HPTS in the three different $\mathrm{pH}$ environments ( $\mathrm{pH} 6,8$ and 10) is relatively flat near $380 \mathrm{~nm}$. These control measurements indicate that the phase dependence arises primarily from differences in the two-photon excitation spectrum and not from intramolecular dynamics.

\section{Discussion}

The MII method is used in this experiment to control twophoton excitation. More specifically, the interference process, which is controlled by the phase introduced in the pulse, suppresses two-photon transitions at specific frequencies within the two-photon bandwidth. ${ }^{2}$ In other words, the interference can be used to tune multiphoton transition probability at specific frequencies. This effect can be envisioned using a thin second harmonic crystal, as shown in the MIIPS traces in Figure 2. Notice that as $\delta$ is scanned the maximum intensity in the second harmonic signal scans as well from longer to shorter wavelengths. ${ }^{1,2}$ By considering this effect together with the twophoton absorption spectrum, we are able to calculate the expected signal using eq 1 with very good agreement.

HPTS is a highly water-soluble, $\mathrm{pH}$-sensitive dye with a $\mathrm{p} K_{\mathrm{a}}$ of $\sim 7.5$ in aqueous solution. ${ }^{20}$ The photochemistry of this system is shown in Figure 4. When in alkaline medium, $\mathrm{pH}>7.5$, the acid-base equilibrium is totally displaced toward the anion form
$\mathrm{pH}>7.5$

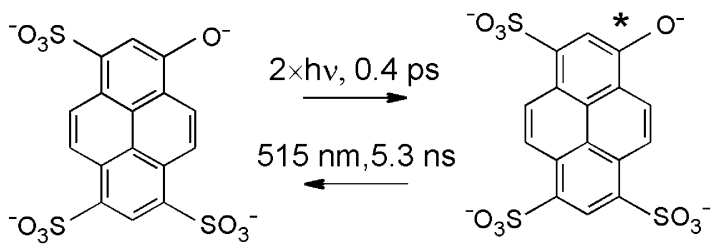

$\mathrm{pH}<7.5$
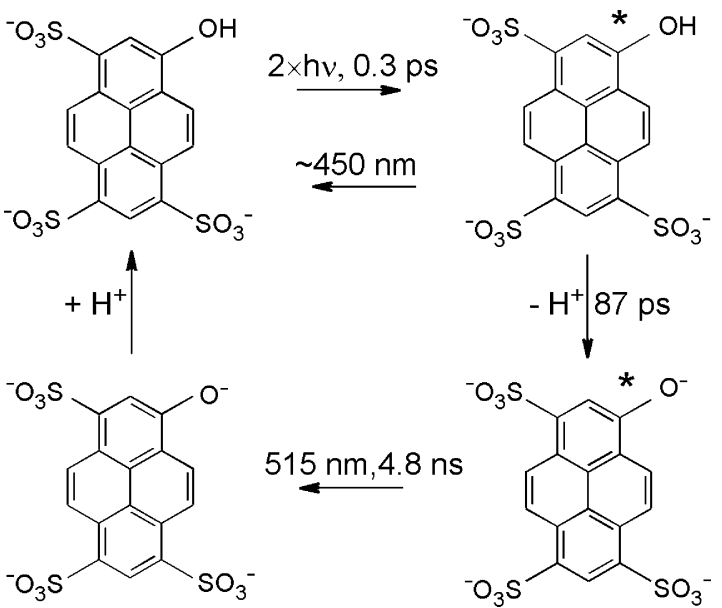

Figure 4. Photochemistry of HTPS in basic $(\mathrm{pH}>7.5)$ and in acid solutions $(\mathrm{pH}<7.5$ ) according to Reference 20 . The equilibrium species in basic solution $\left(3 \mathrm{sPyO}^{-}\right)$upon two-photon excitation reaches an excited state that is solvated within $0.4 \mathrm{ps}$. Fluorescence from $3 \mathrm{sPyO}^{-*}$ has a lifetime of $5.3 \mathrm{~ns}$ and is centered at $515 \mathrm{~nm}$. The equilibrium species in acidic solution (3sPyOH) upon two-photon excitation reaches an excited state that is solvated within 0.3 ps. Fluorescence from $3 \mathrm{sPyOH}^{*}$ is near $450 \mathrm{~nm}$; however, deprotonation to yield $\left(3 \mathrm{sPyO}^{-*}\right)$ takes place within $87 \mathrm{ps}$ and is followed by fluorescence centered at $515 \mathrm{~nm}$ with a $4.8 \mathrm{~ns}$ lifetime.

$\left(3 \mathrm{sPyO}^{-}\right)$of the dye. The electronic character of $3 \mathrm{sPyO}^{-}$ remains unchanged after photoexcitation and corresponds to a singlet-excited state. ${ }^{21}$ Fluorescence from this state undergoes a fast $0.4 \mathrm{ps}$ Stokes shift and has a maximum at $515 \mathrm{~nm}$ and a lifetime of $5.3 \pm 0.1 \mathrm{~ns}^{22}$

In acidic medium, $\mathrm{pH}<7.5$, the conjugate acid form (3sPyOH) dominates in the ground state. Two-photon excitation yields the excited state $\left(3 \mathrm{sPyOH}^{*}\right)$. Fluorescence from this state undergoes a fast 0.3 ps Stokes shift and has a maximum near $450 \mathrm{~nm}$. HPTS has a very high fluorescence quantum yield (almost $100 \%$ in both alkaline and acidic solution, when excited with light of wavelengths shorter than $400 \mathrm{~nm}){ }^{23}$ Proton transfer in the excited state takes place in $87.5 \mathrm{ps},{ }^{21,22}$ to yield the conjugate base $\left(3 \mathrm{sPyO}^{-*}\right)$ (see Figure 4$)$, with a fluorescence maximum at $515 \mathrm{~nm}$ and a lifetime of $4.8 \pm 0.5 \mathrm{~ns}$. Therefore, in both acidic and basic environments, the emission spectrum is unchanged. The nature of the excited-state dynamics involves a three-step mechanism of the proton-transfer reaction, whereby an intermediate state (un-ionized acid form) with significant charge-transfer character converts to the fluorescent excited anionic state. ${ }^{22}$

There has been speculation about the manipulation of intramolecular dynamics of large molecules in condensed phase. In Figure 5, we show the spectrum, phase and time profiles for the shaped pulses with $\delta=\pi / 4$ (dotted line) and $3 \pi / 4$ (dashed line). Notice that both of the pulses show identical time structure. 


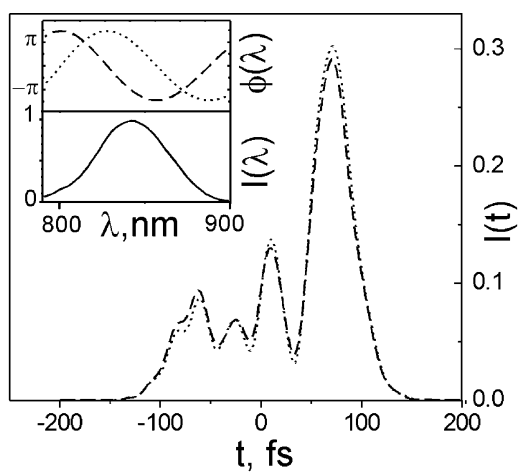

Figure 5. Laser pulse characteristics in the time and spectral domain. The insert shows the measured spectrum of the pulse $I(\lambda)$ and the phase $f(\lambda)$ programmed by the light modulator. The pulses have a Gaussian shape with center wavelength, $\lambda_{0}=842.6$ and $48 \mathrm{~nm}$ fwhm. The temporal profile for two pulses (dotted and dashed lines) is calculated from the experimental parameters for $\delta=1 / 4 \pi$ and $\delta=3 / 4 \pi$, respectively; see text. Notice that the two pulses have the same temporal profile.

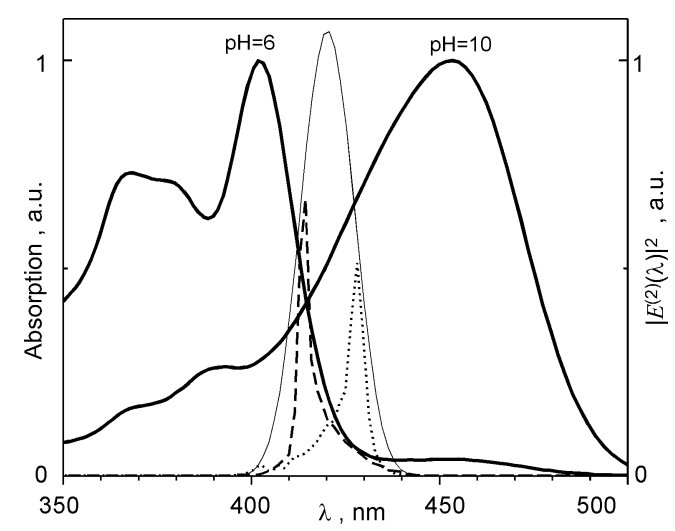

Figure 6. Absorption spectrum of HPTS in acidic and basic solution (bold lines). The thin line is the calculated spectrum of the square of the electric field, proportional to $\left|E^{(2)}(\lambda)\right|^{2}$ of the laser pulse without phase modulation (transform-limited). The square of the electric field of the laser pulse is also calculated for shaped pulses with $\delta=1 / 4 \pi$ (dotted) and $3 / 4 \pi$ (dashed), respectively. The two spectra are very different because of MII (see refs 1 and 2). Notice that, in the frequency domain, it is clear that shaped pulses with $\delta=1 / 4 \pi$ favor two-photon excitation of HPTS in $\mathrm{pH} 10$, whereas pulses with $\delta=3 / 4 \pi$ favor two-photon excitation of HPTS in $\mathrm{pH} 6$.

The periodicity of the structure has an average period of $\sim 50$ fs and lasts for about $200 \mathrm{fs}$. Given the similarities between the two pulses, the control mechanism does not depend on temporal differences. The typical electronic dephasing time of laser dyes in solution is shorter than $\sim 100 \mathrm{fs}$, preventing coherent manipulation by these shaped pulses. Based on these observations and the fact that the simulations, which neglect intramolecular dynamics, are in excellent agreement with the experimental data (especially $\mathrm{pH} 10$ ), we conclude that intramolecular dynamics plays a minimal role in the control that is observed.

Some discrepancy between experiment and simulation is seen for the data obtained under acidic conditions. The simulations were obtained using the measured linear absorption spectrum of HPTS at pH 6 (see Figure 6). It is possible that the twophoton spectrum is significantly different from the one-photon absorption spectrum. Alternatively, fast dynamics may be causing the difference. However, the time scale for proton transfer is much slower than the shaped pulses. The difference between result and simulation for $\mathrm{pH} 6$ will be explored in more detail in a future study.
In Figure 6, we show the absorption spectrum of HPTS at $\mathrm{pH} 6$ and $\mathrm{pH} 10$. The spectrum of the electric field $\mathrm{E}^{2}(t)$ is proportional to $\left|\mathrm{E}^{(2)}(\lambda)\right|^{2}$ and is shown for a transform-limited pulse (thin line) and for phase modulated pulses with $\delta=\pi / 4$ (dotted line) and $3 \pi / 4$ (dashed line). Notice that two-photon transitions at $410 \mathrm{~nm}$ take place preferentially when $\delta=\pi / 4$, favoring probe molecules in a $\mathrm{pH} 10$ environment. Two-photon transitions at $430 \mathrm{~nm}$ take place preferentially when $\delta=3 \pi / 4$, favoring probe molecules in a $\mathrm{pH} 6$ environment. This explanation in the frequency domain is consistent with the observed results and simulations. Note that phase shaping, as shown in our experiments, does not tune the wavelength of the laser pulse. The changes in the two-photon probability for different frequencies arise from the interference, which is controlled by the spectral phase imprinted in the pulses., ${ }^{1,2}$

Recently, two-photon microscopy has gained acceptance for biological imaging applications, given the method's higher resolution, background-free signal, lower background scattering, greatly improved depth of focus, and reduced photon induced damage. ${ }^{24-27}$ Two-photon microscopy has also been valuable in multiple-probe staining, where two-photon transitions excite different probe molecules that emit at different wavelengths, ${ }^{27,28}$ and in functional imaging of living cells. ${ }^{29-32}$ The only drawback to multiphoton microscopy is that selective excitation cannot be realized. Experiments based on dual channel fluorescence detection $^{33,34}$ bring about selective detection, provided very different wavelengths are used, typically green and red. This selectivity cannot be achieved when the reporter molecules have a similar fluorescence spectrum. For example, in the case of HPTS, the fluorescence spectrum is independent of $\mathrm{pH}$.

In Figure 7, we show selective two-photon microscopy images using shaped femtosecond laser pulses. In this experiment, we selectively excite regions that differ in $\mathrm{pH}$ using HPTS as the fluorescent probe. Here selective imaging is achieved with a fluorescent probe that emits at $515 \mathrm{~nm}$ in both acid and basic $\mathrm{pH}$, making it incompatible with dual fluorescence detection. Figure 7A shows an image obtained with transform-limited pulses (time duration is $23 \mathrm{fs}$ at $842.6 \mathrm{~nm}$ ), where fluorescence from HPTS under both acidic and basic conditions occurs without selectivity. However, when laser pulses are optimally shaped for selective excitation, fluorescence switches on and off depending on whether the chemical environment of the molecule is acidic or basic. The pulses were shaped with $\alpha=$ $1.5 \pi \mathrm{rad}$ and $\gamma=20 \mathrm{fs}$. For the acidic probe, $\delta$ was optimized to $0.75 \pi \mathrm{rad}$; for the basic probe, $\delta$ was $0.25 \pi \mathrm{rad}$. Figure $7 \mathrm{~B}, \mathrm{C}$ shows images obtained under these conditions. Very high contrast is observed between the two different $\mathrm{pH}$ regions.

Pulse shaping and, in particular, the use of MII provide a number of advantages for multiphoton microscopy. (a) A very short pulse laser is set up once and optimized once (no subsequent tweaking). (b) The short pulse duration has the advantages of higher multiphoton excitation efficiency and broader bandwidth. (c) As the phase is optimized for selective excitation, maximum efficiency is maintained; there is no need for tuning, realigning, tweaking, compensating for chirp, and renormalizing. (d) The system is computer-controlled and can take advantage of phase compensation at the sample using MIIPS as described here. (e) MII can be used to control twoor three-photon excitation. (f) During the selective excitation experiments, the intensity and spectrum of the laser remain constant; therefore, one-photon processes such as absorption, reflection, and scattering remain constant as well. (g) Selective photochemistry can be realized through shaped pulse excitation and this can be used for selective multiphoton microlithography. 


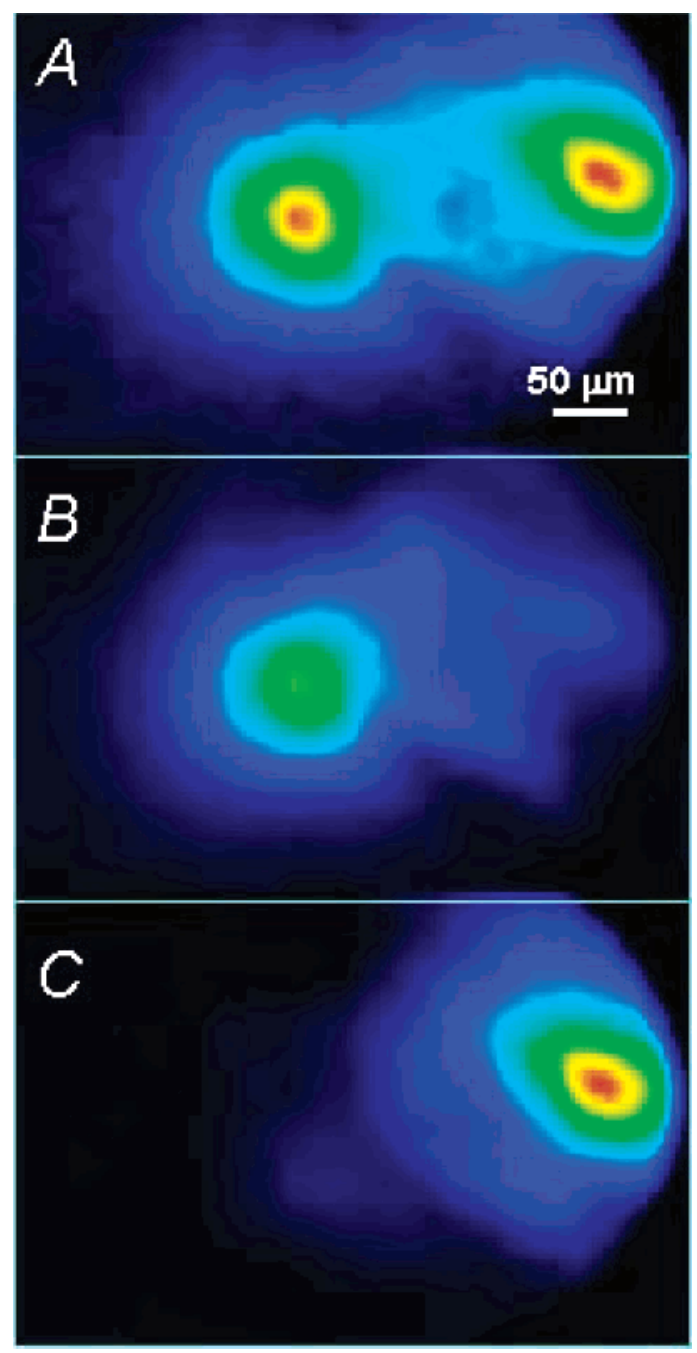

Figure 7. Experimental demonstration of two-photon selective microscopy. The HPTS-labeled sample being imaged has an acidic (left side of frame, at $\mathrm{pH}$ 6) and a basic (right side of frame, at $\mathrm{pH} 10$ ) region. Images were obtained with (A) 23-fs transform-limited pulses centered at $842 \mathrm{~nm}$ and (B and C) phase shaped pulses optimized for selective excitation. The image is presented in false color; the fluorescence wavelength is centered at $515 \mathrm{~nm}$.

\section{Conclusion}

Selective two-photon excitation is achieved by optimizing the overlap between the power spectrum of the electric fieldsquared (by spectral phase modulation) and the two-photon absorption spectrum of the molecule. Phase modulation of ultrashort pulses through multiphoton intrapulse interference can be used to probe chemical changes that take place within cells as they respond to external changes in their environment. The effect can be further enhanced using even shorter (larger bandwidth) fs pulses. The quantification of changes in intracellular ion concentration can be accomplished with the use of fluorescent indicator dyes ${ }^{35}$ based on shifts in their two-photon excitation spectrum. MII has demonstrated applicability in the study of biochemical phenomena, and this is validated by its significant role in selective microscopy. ${ }^{3}$ Practically all of the cellular processes can potentially be affected by changes in intracellular $\mathrm{pH}$. The absorbance and/or emission properties of fluorescent dyes are affected by their environment, including solvent, $\mathrm{pH}$, and conjugation to other macromolecules (e.g., nucleic acids). Changes in intracellular $\mathrm{pH}$ are often one of the responses of cells to externally applied agents, including growth factors and pathogens. ${ }^{36}$ The principles of MII can be similarly applied to other biologically relevant local environment parameters, like sodium or calcium ion concentration.

Acknowledgment. This research was funded by the Chemical Sciences, Geosciences and Biosciences Division, Office of Basic Energy Sciences, Office of Science, U.S. Department of Energy. M.D. is a Camille Dreyfus Teacher-Scholar. We thank Prof. Gary Blanchard for allowing us to use his microscope for the imaging experiment.

\section{References and Notes}

(1) Walowicz, K. A.; Pastirk, I.; Lozovoy, V. V.; Dantus, M. J. Phys. Chem. A 2002, 106, 9369.

(2) Lozovoy, V. V.; Pastirk, I.; Walowicz, K. A.; Dantus, M. J. Chem. Phys. 2003, 118, 3187

(3) Pastirk, I.; Dela Cruz, J. M.; Walowicz, K. A.; Lozovoy, V. V.; Dantus, M. Opt. Express 2003, 11, 1695.

(4) Cerullo, G.; Bardeen, C. J.; Wang, Q.; Shank, C. V. Chem. Phys. Lett. 1996, 262, 362 .

(5) Bardeen, C. J.; Yakovlev, V. V.; Squier, J. A.; Wilson, K. R. J. Am. Chem. Soc. 1998, 120, 13023. 414,57

6) Brixner, T.; Damrauer, N. H.; Niklaus, P.; Gerber, G. Nature 2001 ,

(7) Herek, J. L.; Wohlleben, W.; Cogdell, R. J.; Zeidler, D.; Motzkus, M. Nature 2002, 417, 533 .

(8) Rice, S. A. Nature 2001, 409, 422.

(9) Bardeen, C. J.; Rosenthal, S. J.; Shank, C. V. J. Phys. Chem. A 1999, 103, 10506

(10) Judson, R. S.; Rabitz, H. Phys. Rev. Lett. 1992, 68, 1500.

(11) Bardeen, C. J.; Yakovlev, V. V.; Wilson, K. R.; Carpenter, S. D.; Weber, P. M.; Warren, W. S. Chem. Phys. Lett. 1997, 280, 151.

(12) Assion, A.; Baumert, T.; Bergt, M.; Brixner, T.; Kiefer, B.; Seyfried, V.; Strehle, M.; Gerber, G. Science 1998, 282, 919.

(13) Levis, R. J.; Menkir, G. M.; Rabitz, H. Science 2001, 292, 709.

(14) Broers, B.; Vandenheuvell, H. B. V.; Noordam, L. D. Opt. Commun. 1992, 91,57

(15) Meshulach, D.; Silberberg, Y. Nature 1998, 396, 239.

(16) Buist, A. H.; Muller, M.; Ghauharali, R. I.; Brakenhoff, G. J.; Squier, J. A.; Bardeen, C. J.; Yakovlev, V. V.; Wilson, K. R. Opt. Lett. 1999, 24, 244.

(17) Weiner, A. M. Rev. Sci. Instr. 2000, 71, 1929.

(18) Lozovoy, V. V.; Pastirk, I.; Dantus, M. Opt. Lett. 2003, submitted.

(19) Trebino, R.; DeLong, K. W.; Fittinghoff, D. N.; Sweetser, J. N.; Krumbugel, M. A.; Richman, B. A.; Kane, D. J. Rev. Sci. Instr. 1997, 68, 3277.

(20) Wolfbeis, O. S.; Furlinger, E.; Kroneis, H.; Marsoner, H. Fresenius Z. Anal. Chem. 1983, 314, 119 .

(21) Tran-Thi, T. H.; Gustavvson, T.; Prayer, C.; Pommeret, S.; Hynes, J. T. Chem. Phys. Lett. 2000, 329, 421.

(22) Tran-Thi, T. H.; Prayer, C.; Millie, P. H.; Uznanski, P.; Hynes, J. T. J. Phys. Chem. A 2002, 106, 2244.

(23) Schulman, S. G.; Vincent, W. R.; Underberg, W. J. M. J. Phys. Chem. 1981, 85, 4068 .

(24) Denk, W.; Strickler, J. H. Science 1990, $248,73$.

(25) Denk, W. J. Biomed. Opt. 1996, 1, 296.

(26) Konig, K. J. Microsc. (Oxford) 2000, 200, 83.

(27) So, P. T. C.; Dong, C. Y.; Masters, B. R.; Berland, K. M. Аnnu. Rev. Biomed. Eng. 2000, 2, 399.

(28) Dunn, K. W.; Sandoval, R. M.; Kelly, K. J.; Dagher, P. C.; Tanner, G. A.; Atkinson, S. J.; Bacallao, R. L.; Molitoris, B. A. Am. J. Physiol.Cell Physiol. 2002, 283, C905.

(29) Wang, J. W.; Wong, A. M.; Flores, J.; Vosshall, L. B.; Axel, R. Cell 2003, 112, 271.

(30) Oertner, T. G. Exp. Physiol. 2002, 87, 733.

(31) Kasischke, K. A.; Vishwasrao, H.; Heikal, A. A.; Webb, W. W. Biophys. J. 2002, 82, 2420.

(32) Rose, C. R.; Kovalchuk, Y.; Eilers, J.; Konnerth, A. Pflugers Arch. 1999, 439, 201

(33) Winkler, T.; Kettling, U.; Koltermann, A.; Eigen, M. Proc. Natl. Acad. Sci. U.S.A. 1999, 96, 1375.

(34) Rarbach, M.; Kettling, U.; Koltermann, A.; Eigen, M. Methods 2001, 24, 104.

(35) Haugland, R. P.; Johnson, I. D. Intracellular Ion Indicators. In Fluorescent and Luminescent Probes; 2nd ed.; Mason, W. T., Ed.; Academic Press: New York, 1999; p 647.

(36) Putnam, R. W. Intracellular pH Regulation. In Cell Physiology Source Book, 2nd ed.; Sperelakis, N., Ed.; Academic Press: New York, 1996; p 738. 